\title{
Incidence de pluies exceptionnelles sur un aquifère hétérogène côtier (M'nasra, Maroc)
}

\author{
A. RHIDOUANI ${ }^{1,2}$, I. IDRISSI ALAMI ${ }^{1,3^{*}}$, M. ADDOU ${ }^{3}$ et I.B.N. AHMED ${ }^{2}$ \\ ${ }^{1}$ Laboratoire Régionale de l'Office National de l'Eau Potable, Kenitra, Maroc. \\ ${ }^{2}$ Laboratoire de Synthèse Organique, Faculté des Sciences de Kenitra BP133, Maroc. \\ ${ }^{3}$ Laboratoire D'optoélectronique et de physico-chimie des matériaux, URAC-14, Université Ibn Tofail, \\ Faculté des Sciences Kenitra, Maroc. \\ *Auteur correspondant, E-mail: idrissialami_ibtissam@yahoo.fr
}

\section{RESUME}

Les eaux souterraines qui représentent les seules ressources en eau de la M'nasra côtière, sont minéralisées : intrusion marine, évaporation, recyclage des eaux d'irrigation chargées en sels et l'utilisation abusive des engrais. L'évolution spatio-temporelle de la pollution nitrique de cette nappe phréatique a été suivie sur 159 puits, objective en trois périodes de campagne éclatées : 2003, 2007 et 2010 qui inclue une période exceptionnellement pluvieuses. Une comparaison par apport à l'état de la nappe en 1993, date de début de la surexploitation des eaux souterraines, a été faite. Les suivis ont montré qu'à la suite des Pluies importantes de la période de campagne 2009/2010, on a pu observer une dégradation de la qualité de la nappe aussi bien au niveau ponctuel qu'au niveau spatial, en particulier dans la partie Nord de la zone étudiée (au nord de la ligne de cordonnée $\mathrm{Y}=430000 \mathrm{~m}$ ). Les résultats de cette étude sont présentés sous forme de cartes thématiques par le SIG.

(C) 2012 International Formulae Group. All rights reserved.

Mots clés: Aquifère côtier, inondation, pollution nitrique, nappe phréatique, M'nasra, Maroc.

\section{INTRODUCTION}

Les études récentes (Ibnoussina et al., 2006; Idrissi et al., 2007; Idrissi, 2009; Idrissi et al., 2010a; et Idrissi et al., 2010b ; Idrissi et al., 2011) ont montré que les problèmes posés par l'exploitation d'un aquifère côtier sont complexes.

En effet, un des problèmes importants auquel fait face l'agriculture d'aujourd'hui est la pollution des eaux souterraines par l'azote nitrique N-NO3, surtout celle due aux applications élevées des engrais azotés dans la production intensive des cultures. Malheureusement, avec l'évolution démographique et l'industrialisation de l'agriculture, les quantités d'engrais azotés utilisées ne cessent d'accroître. Les taux d'application d'engrais azotés ont atteint 32 270 kilogrammes de N.ha-1 donnant une moyenne de $117 \mathrm{~kg}$ N/ha (SCET Maroc) pour la production des haricots secs dans la région de M'nasra à titre d'exemple. En outre, la nappe de la zone côtière, très productive et facilement accessible, est exploitée d'une manière intensive pour l'irrigation par des pompages privés (Zeraouli et al., 2001; Bricha et al., 2007). Le nombre de puits creusés dans la nappe dépasse 20000 . La forte 
perméabilité du sol et du sous-sol et la faible profondeur de la nappe sont très vulnérables à la pollution agricole diffuse $\left(\mathrm{N}-\mathrm{NO}_{3}\right)$.

La situation de la nappe de M'nasra, qui fait l'objet de notre étude, est identique à celle de certaines nappes côtières, notamment en Algérie (Boudjadja et al., 2003), en Côte d'Ivoire (Goula et al., 2007) et en Tunisie par (Hatira et al., 2007).

L'Objectif de ce travail est d'étudier le comportement et la qualité physico-chimique de la nappe phréatique soumise aux pluies exceptionnelles de la campagne 2009/2010 et d'identifier les secteurs les plus sensibles aux recharges pluviales.

\section{MATERIEL ET METHODES}

Cadre géographique, socio-économique et climatique

La M'nasra constitue la zone la plus agricole de la plaine de Gharb. Formant la continuité géographique de la plaine du Gharb le long de l'océan atlantique. L'aire de la zone dite M'nasra s'étend sur une superficie de 600 $\mathrm{Km}^{2}$ entre la ville de Kenitra au Sud, l'Oued Sebou prolongé par la ligne parallèle passant par Sidi Allal Tazi à l'Est, la Merja Zega à proximité de Moulay Bouselham au Nord (Figure 1). Elle bénéficie d'une superficie agricole utile de l'ordre de $70 \%$ de la superficie totale, ce qui lui confère une production agricole assez importante de l'ordre de $12 \%$ de la production nationale (Zeraouli et al, 2001). Elle s'est développée une forte activité de marâichage (soit une superficie de $8398 \mathrm{Ha}$ ) et de primeurs (soit une superficie de $17985 \mathrm{Ha}$ ), accompagnées d'un essor démographique important. Selon le recensement général de la population et de l'habitat, la population totale de la zone du M'nasra est passée de 118867 en 1994 à 144841 en 2004, soit un taux d'accroissement démographique de $1.99 \%$ (presque identique au taux moyen observé au niveau national).

Le climat de la région du Gharb est de type méditerranéen avec influence océanique.
Il varie progressivement de l'étage subhumide à influence océanique dans la zone côtière (le site étudié) à un climat semi-aride tempéré à l'Est de la plaine. La région du Gharb reçoit annuellement des précipitations moyennes, d'environ $500 \mathrm{~mm}$, marquées par une pluviométrie plus élevée dans le Nord jusqu'à plus de $900 \mathrm{~mm}$, d'environ $600 \mathrm{~mm}$ dans la zone côtière et relativement faible dans le Sud Est vers Sidi Kacem qui descend jusqu'à 400 mm (ORMVAG, 2010). Ces pluviométries témoignent d'une réelle variabilité géographique et sont marquées par une double irrégularité annuelle et périodique (Figure 2 et 3).

La pluviométrie moyenne enregistrée entre septembre et la première décade de mars 2010 a atteint $746 \mathrm{~mm}$. Cette pluviométrie est la plus importante de toutes les précipitations annuelles depuis les années 60 (ORMVAG, 2010). En général, les précipitations s'étalent sur 7 mois, entre mi-Octobre et mi-Avril, en moyenne sur 60 à 70 jours (1 jour sur 5) avec un maximum principal en Décembre et un maximum secondaire généralement en Mars. La comparaison des précipitations mensuelles de la campagne 2009/2010, avec celles de la campagne précédente 2008/2009 et la moyenne des 30 dernières années est donnée sur la Figure 4:

Le début de la campagne 2009/2010 a été marqué par une période de sécheresse avec des précipitations très faibles jusqu'au 13 Décembre qui marque le début d'une période de fortes précipitations concentrées dans le temps (206 mm en 17 jours).

Les précipitations mensuelles, à partir du mois de Décembre, dépassent largement la moyenne des 30 dernières années (plus que le double), ainsi que les précipitations de la campagne précédente (avec plus de 5\%) dont les inondations et les stagnations d'eau ont touché $138.000 \mathrm{Ha}$. Ces précipitations dépassent celles de 1995/96 de 20\% (avant la mise en eau du barrage Al wahda) dont les superficies submergées ont atteint $140.000 \mathrm{Ha}$ 
Les précipitations de 2009/2010 sont exceptionnelles du fait qu'elles se sont concentrées entre mi-Décembre et début Mars d'une manière continue, ce qui n'a pas permis l'évacuation des eaux stagnées auxquelles se sont rajoutées les eaux d'inondations.

La surface irriguée atteint 39686 ha (DPA, 2006). Les retours d'irrigation vers la nappe, estimés à $25 \%$ sur des lysimètres (DRPE, 2006) et chargés en sels issus surtout $\mathrm{du}$ lessivage, constituent une source potentielle de dégradation de la qualité des eaux souterraines (Lhadi et al., 1993)

La pollution des eaux par les nitrates est aujourd'hui un problème planétaire. L'origine et les effets néfastes de la contamination nitrique ont été largement étudiés (Laurence, 2007 ; Laurianne, 2008).

Les effets toxiques des ions nitrates, sur la santé humaine et le milieu environnemental, sont principalement la méthémoglobinémie, la cancérigènité causée par les nitrosamines, et l'eutrophisation (L'hirondel, 2002) ; (Leclerc, 2008 et Lenntech, 2008).

\section{Echantillonnage et analyse}

Pour l'échantillonnage, l'eau est prélevée directement du réseau d'irrigation pour les puits qui sont équipés de pompes de refoulement. Pour ceux qui ne sont pas équipés, le prélèvement est effectué à l'aide d'un récipient métallique leste. L'ensemble des prélèvements a été effectué dans des flacons en polyéthylène ou en verres borosilicate. Les échantillons ont été soigneusement étiquetés et conservés à $+4{ }^{\circ} \mathrm{C}$, jusqu'à leurs arrivées au laboratoire selon les normes de la loi Afnor NFT. 90-031. Parue en 1977. Les nitrates ont été mesurés par spectrométrie d'absorption moléculaire selon le protocole développé par Rodier (1984). Après réduction en nitrite sur cadmium, les nitrates sont quantitativement réduits en nitrites par cadmium $(\mathrm{Cd})$ recouvert d'une couche de cuivre après traitement au sulfate de cuivre. Les nitrites produits, forment avec l'amino-4-benzène sulfonamide un composé diazoïque, lequel couplé avec la N-(Naphty-1) diamine-1,2-éthane donne un complexe rose susceptible d'être mesuré au spectrophotomètre à la longueur d'onde de $540 \mathrm{~nm}$.

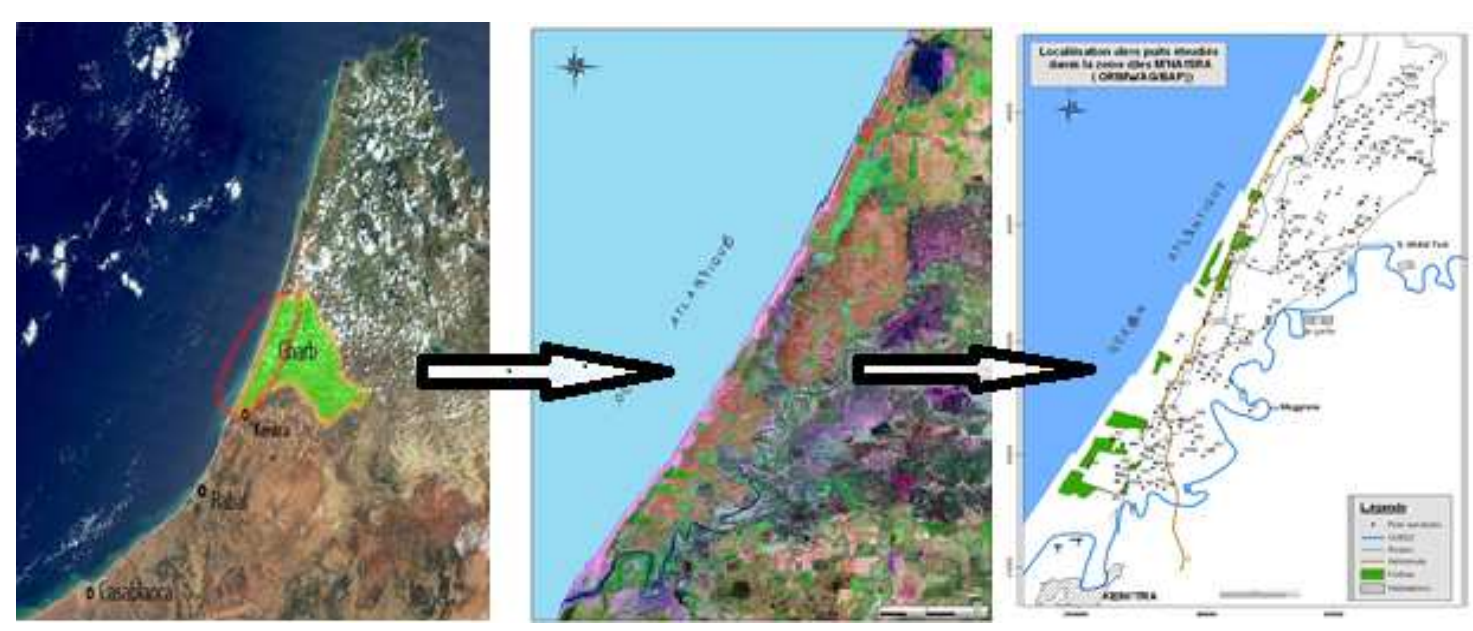

Figure 1: Photo aérienne de la zone M'nasra et localisation des 159 puits étudiée (ORMVAGDDA /SEA/BAP). 


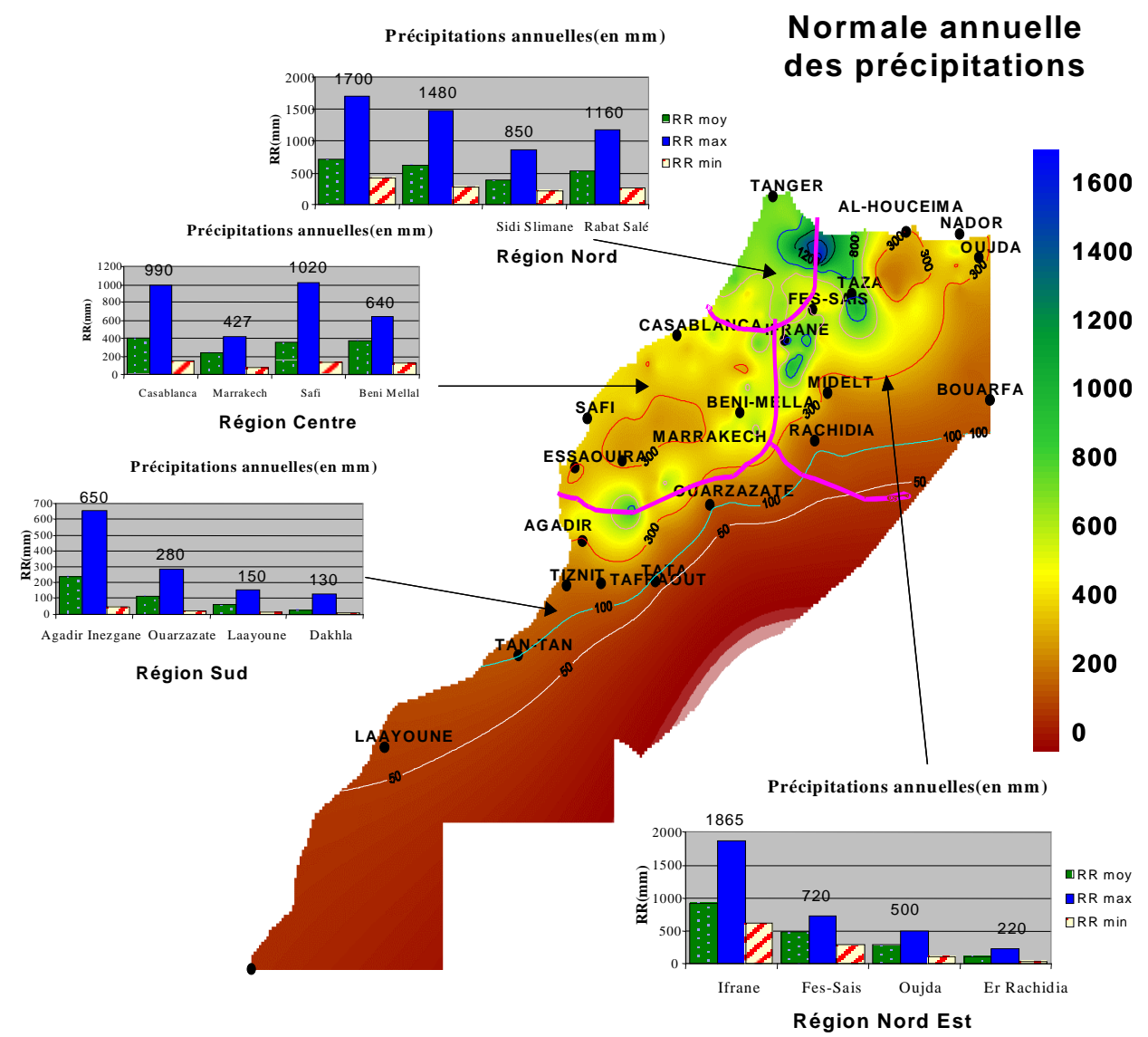

(Secrétariat d'Etat auprès du Ministère de l'Aménagement du Territoire, de l'Eau et de l'Environnement, chargé de l'eau)

Figure 2: Variabilité spatiale des ressources en eau.

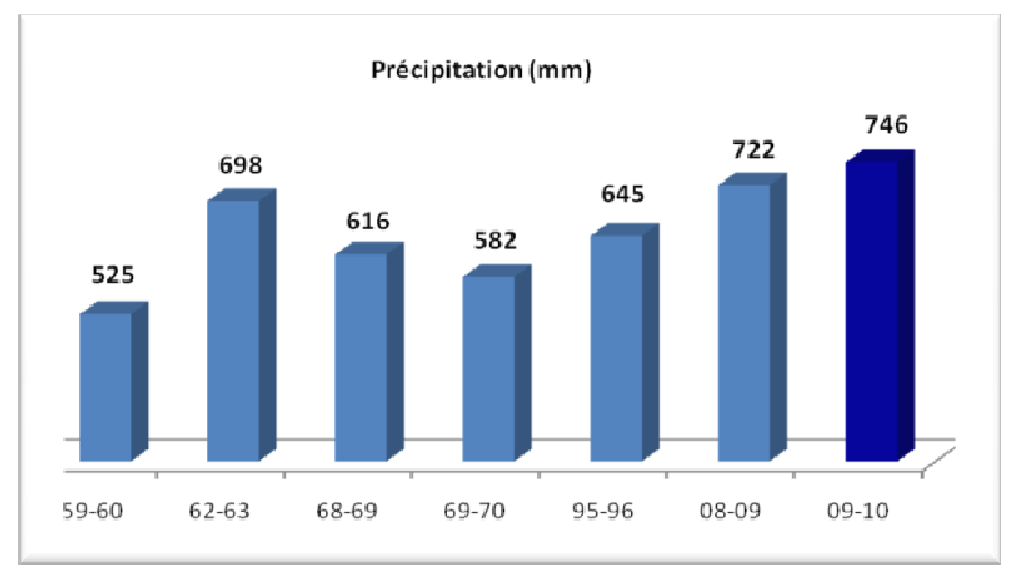

Figure 3: Précipitations du début septembre au 10 Mars (ORMVAG D.D.A/S.E.D 1). 


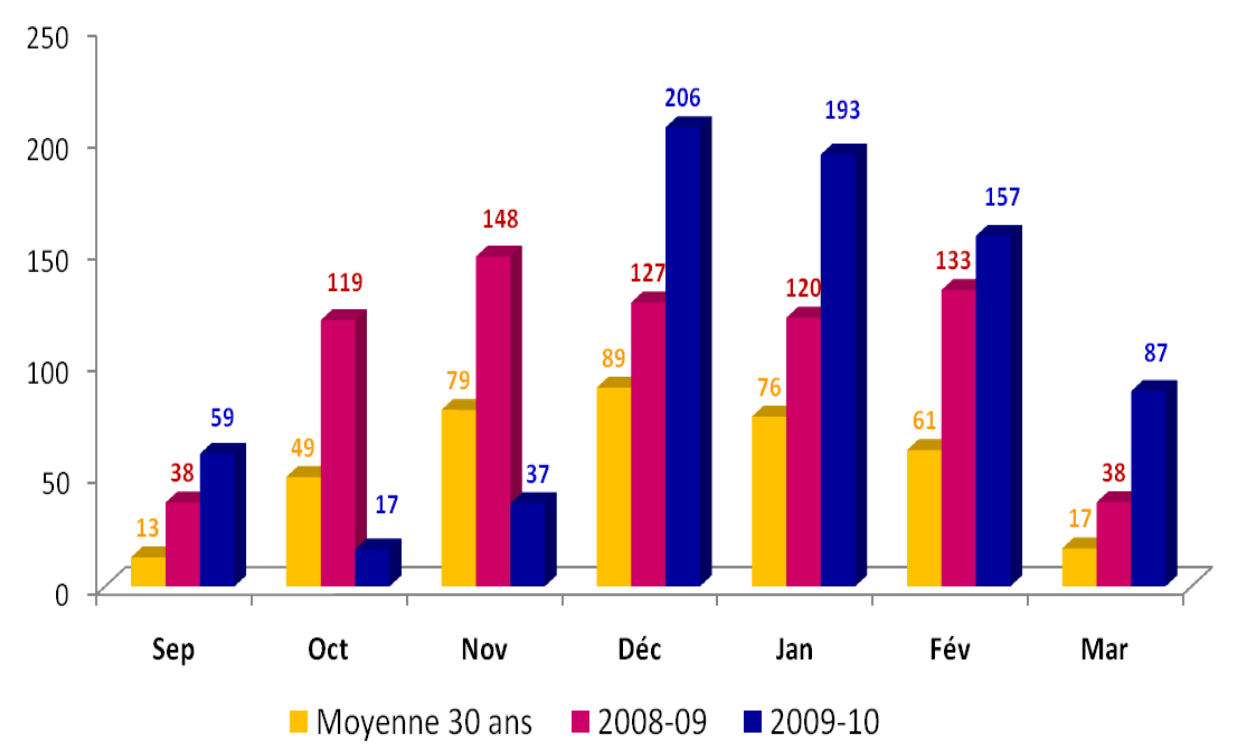

Figure 4 : Précipitations mensuelles (mm) (ORMVAG D.D.A/S.E.D 1).

\section{RESULTATS ET DISCUSSION}

Mise en évidence des pluies exceptionnelles de la campagne (2009-2010) d'après l'évolution spatio-temporelle de la pollution nitrique

Etat de la nappe avant les pluies exceptionnelles (1993/2003/2007)

La contamination des eaux souterraines marocaines par les nitrates est un phénomène qui touche presque la totalité des bassins hydrauliques au Maroc. La pollution nitrique de la nappe phréatique de la zone côtière du Gharb du Maroc (M'nasra) a été constatée depuis 1993 par l'Office Régional de Mise en Valeur Agricole du Gharb. En effet, nous avons mesuré les nitrates de 159 puits de la zone M'nasra pour les périodes de campagne 2003 et 2007, correspondant aux mêmes puits étudiés en 1993 par l'ORMVAG. Selon l'OMS, la teneur en nitrates des eaux potables ne doit pas dépasser la concentration de 50 $\mathrm{mg} / \mathrm{L}$. Ceci nous a permis de réaliser une répartition des 159 puits en cinq classes (Tableau 1).

Analyse des fréquences des puits selon la concentration des nitrates

Nous avons évoqué la fréquence des puits en fonction de la concentration des nitrates. Ces pourcentages, sont donnés dans la Figure 5. Les résultats sont représentés sous forme de classes. Nous remarquons un abattement de la fréquence des puits. Nous représentons sur la Figure 6, les puits possédant une concentration des nitrates supérieure à $50 \mathrm{mg} / \mathrm{L}$ relevée pendant les mêmes périodes d'échantillonnage. Par conséquence, cette fréquence avoisine : $73,6 \%, 82,39 \%$ et $83,02 \%$ respectivement pendant les périodes de mesure 1993, 2003 et 2007.

Il apparaît une diminution du taux de pourcentage des puits dont la qualité est bonne et par conséquent une augmentation de la fréquence des puits dont la qualité est mauvaise soit de $0,63 \%, 8,79 \%$ et $09,42 \%$, 
enregistré respectivement entre les périodes de campagne 2003 et 2007 ; 1993 et 2003 et entre 1993 et 2007.

Ces résultats convergent vers une pollution nitrique de la nappe de M'nasra entre les périodes de campagnes 1993 et 2007. Ceci peut s'expliquer par l'intensification agricole qui prend une ampleur de plus en plus importante, notamment des apports abusifs d'engrais azotés conjugués à un mode d'irrigation inadapté.

\section{Etudes statistiques des résultats obtenus des nitrates}

Les principaux résultats montrent que les moyennes générales de la concentration des nitrates relevée pendant les périodes de campagne 1993, 2003 et 2007 sont respectivement de $107,2 \mathrm{mg} / \mathrm{L}, 120,4 \mathrm{mg} / \mathrm{L}$ et $146,5 \mathrm{mg} / \mathrm{l}$; soit une augmentation de 13,2 $\mathrm{mg} / \mathrm{L}$ entre 1993 et 2003, 26,1 mg/L entre 2003 et 2007 , et une augmentation de 39,3 $\mathrm{mg} / \mathrm{L}$ entre 1993 et 2007.

En faisant la comparaison de la moyenne générale de la concentration des nitrates calculé, nous pouvons constater clairement que cette moyenne augmente en fonction du temps. Pour savoir la signification de cette augmentation du point de vue statistique, et afin de mieux encercler la localisation de la pollution nitrique, la zone étudiée a été subdivisée en deux parties : une zone Nord appelée zone A et une zone Sud appelée zone B (Figure 7). Les deux zones sont séparées par la ligne de coordonnée y = $430000 \mathrm{~m}$.

Zone A : C'est la zone située au Nord de la ligne de cordonnée $\mathrm{Y}=430000 \mathrm{~m}$, elle englobe $72 \%$ de l'ensemble des puits étudiés. Zone B : C'est la zone située au Sud de la ligne de cordonnée $\mathrm{Y}=430000 \mathrm{~m}$, elle englobe $\quad 28 \%$ de l'ensemble des puits étudiés.

Les résultats de l'analyse de la variance des 159 puits constituants les deux zones sont présentés dans le Tableau 2. L'analyse de ce tableau, permet de tirer les constatations suivantes :

L'analyse de la variance des 159 puits montre que la dégradation de la qualité nitrique de la nappe M'nasra est observée essentiellement au Nord (de ligne de cordonnée Y=430 000 m) que dans le Sud. Ceci peut être expliqué par deux causes essentielles :

*L'intensification accrue des cultures sous serre (superficie totale de 1361 ha) (Figure 8) dans la partie Nord $(\approx 74 \%)$ que dans la partie Sud $(\approx 26 \%)$

* L'organisation des écoulements souterrains se scinde en deux parties : La partie s'étendant au Nord du barrage de garde (zone A), et la partie s'étendant entre cet ouvrage et la ville de Kenitra (zone B) (Figure 9).

Dans la partie septentrionale, les courbes piézométriques forment un bombement dont l'axe N-S correspond à une ligne de partage des eaux donnant lieu à deux directions d'écoulement, l'une vers l'océan et l'autre vers Oued Sebou. La ligne de partage des eaux est régie par la ligne de crête de la surface piézométrique au droit du secteur dunaire interne. A l'Ouest, l'écoulement se fait en direction de l'Océan. Par ailleurs, c'est dans la zone Nord où nous rencontrons les dunes et les replats dunaires intérieurs à relief peu mouvementé, élevées de 5 à 20 m d'altitude. Ainsi, elles correspondent à la zone la plus large du domaine sableux et dont la nappe phréatique est très proche de la surface (3 à $13 \mathrm{~m}$ ), ce qui explique l'exploitation intensive actuelle. En conséquence, cette étude a soulevé une pollution nitrique plus accentuée dans le Nord que dans le Sud de la zone M'nasra. Cette dégradation a été déjà notée en comparant la situation de 1993 avec celle de 2003 et s'est confirmée davantage avec la comparaison des données en 2007. En revanche, la dégradation s'est faite moins 
sentir au Sud, celle-ci n'a été notée que lorsque nous avons comparé les données de 2007 avec 1993. Alors, nous pouvons conclure que nous avons deux dynamiques de dégradation différentes.

Etude spatiale des résultats obtenus des nitrates

Afin d'exploiter au mieux nos résultats d'analyse, nous avons exploité le système d'information Géographique SIG-Arcview et son extension spatiale analyste en vue d'élaborer des cartes thématiques de la concentration des nitrates pendant les campagnes de mesure 1993, 2003 et en 2007 (Figure 10).

L'analyse grossière de ces cartes, a confirmé une pollution nitrique plus accentuée dans le Nord que dans le Sud de la zone M'nasra. Nous pouvons constater clairement une diminution de la superficie occupée par les classes $\mathrm{C} 1, \mathrm{C} 2$ et $\mathrm{C} 3$ respectivement pendant les périodes de campagne 1993, 2003 et 2007. En revanche, les superficies occupées par les classes C4 et C5 ont connu une augmentation évolutive et répartie pendant les mêmes périodes de campagne. Pour mieux comprendre cette variation, nous les avons exprimés en pourcentages. Nous avons résumé les résultats obtenus dans le Tableau 3.

Ces résultats montrent que l'espace couvert par la nappe dont la concentration en nitrate est supérieure à $50 \mathrm{mg} / \mathrm{L}$, a gagné du terrain soit respectivement de $5600 \mathrm{Ha}$ et 1938 Ha entre les périodes de campagnes 1993, 2003, et 2003, 2007. Par conséquent, nous avons eu une superficie de 7538 Ha dont la nappe M'nasra a connu une pollution par les nitrate entre les périodes de mesures 1993 et 2007. Ceci confirme la dégradation de la qualité de la nappe aussi bien au niveau ponctuel qu'au niveau spatial, surtout dans la partie Nord de la zone. Etat de la nappe après les pluies
exceptionnelles (2010)

En juin 2010, nous avons repris la mesure des nitrates effectuée pendant les années 1993, 2003 et 2007.

Le Tableau 4 indique les niveaux moyens de la concentration des nitrates, les niveaux Max, Min, et les Ecart-types calculés au niveau des 159 puits en 1993, 2003,2007 et 2010.

En faisant la comparaison de la moyenne générale de la concentration des nitrates calculée, nous pouvons constater clairement que cette moyenne a continué à augmenter pendant la période de campagne 2010. Pour voir la signification de cette augmentation, nous avons effectué une étude de la variance. Les résultats de l'analyse de la variance des 159 puits et de chaque zone A et B sont reportés dans le Tableau 5:

L'analyse statistique des résultats a relevé qu'il y a eu une continuation de la dégradation de la qualité de la nappe de M'nasra, surtout dans la zone Nord. Après les pluies de 2010, on a constaté une augmentation de la concentration des nitrates.

Exploitation des données des nitrates par étalement spatiale

Pour étudier et comparer la répartition spatiale des zones polluées de la zone M'nasra, nous avons élaboré la carte thématique des concentrations des nitrates calculées en 2010, et nous l'avons comparé avec les cartes thématiques de la concentration des nitrates mesurée pendant les périodes de campagnes d'échantillonnage 1993, 2003 et 2007.

L'analyse grossière des cartes (Figure 11), a toujours confirmé une pollution nitrique plus accentuée dans le Nord que dans le Sud de la zone M'nasra. Afin d'exploiter la superficie occupée par chaque classe de la concentration des nitrates, nous l'avons relaté 
sous forme d'un histogramme représenté par la Figure 12

Nous pouvons constater clairement que les superficies occupées par les classe C1, C2 et $\mathrm{C} 3$ continueront à diminuer respectivement pendant la période de campagne 2010. En outre, les superficies occupées par les classes $\mathrm{C} 4$ et $\mathrm{C} 5$ continueront à augmenter pendant la même période de prélèvement. Pour mieux exprimer cette variation, nous l'avons exprimé en pourcentages. Les résultats obtenus sont donnés dans le Tableau 6.

Pour comparer la variation de la concentration des nitrates au niveau des différentes classes, nous les avons exposé sous forme d'un histogramme représenté dans la Figure 13.

Nous pouvons donc constater que :

* L'espace couvert par la nappe dont la concentration nitrique est inférieure à $50 \mathrm{mg} / \mathrm{l}$ perdra du terrain soit $483 \mathrm{Ha}$ et 1593 ha respectivement entre les périodes de campagnes 2007 et 2010. En conséquence, cette fréquence avoisine $20 \%, 15 \%, 11 \%$ et $07 \%$, respectivement pendant les périodes de mesure 1993, 2003, 2007 et 2010.

* L'espace couvert par la nappe dont la concentration nitrique est deux fois la norme, perdra du terrain en fonction du temps. En conséquence cette fréquence avoisine : $27 \%$,
$24 \%, 16 \%$ et $12 \%$, respectivement pendant les périodes de mesure 1993, 2003,2007, 2010.

* L'espace couvert par la nappe dont la concentration nitrique est trois fois la norme, perdra du terrain en fonction du temps. En conséquence cette fréquence avoisine : $21 \%$, $22 \%, 17 \%$ et $11 \%$, respectivement pendant les périodes de mesure 1993, 2003, 2007 et 2010.

* L'espace couvert par la nappe dont la concentration nitrique est supérieure quatre fois a la norme autorisée C4 (comprise entre 150 et $250 \mathrm{mg} / \mathrm{L}$ ) semblera gagner du terrain surtout dans la partie nord, entre 1993 et 2007 mais, elle a récupéré son terrain perdu en 2010.

* L'espace couvert par la nappe dont la concentration nitrique est supérieure cinq fois à la norme autorisée C5 (> $250 \mathrm{mg} / \mathrm{l})$ a gagné du terrain surtout dans la partie nord en passant de $12 \%$ à $52 \%$ en 2010.

Ces résultats confirment qu'après les pluies exceptionnelles de la période de prélèvement 2010, la qualité de la nappe de M'nasra a continué à se dégrader. En effet, cette nappe est très vulnérable à la pollution agricole diffuse (N-NO3) du fait de la forte perméabilité du sol et du sous-sol et de sa situation à faible profondeur.

Tableau 1: Classification des puits en fonction de la teneur en nitrate.

\begin{tabular}{lcc}
\hline Classe & Teneur en nitrates $\mathbf{~ T i})$ en $\mathbf{( m g} / \mathbf{L})$ & Qualité \\
\hline $\mathrm{C} 1$ & $\mathrm{Ti} \leq 50$ & Bonne \\
\hline $\mathrm{C} 2$ & $50<\mathrm{T} \mathbf{i} \leq 100$ & \\
$\mathrm{C} 3$ & $100<\mathrm{Ti} \leq 150$ & Mauvaise \\
$\mathrm{C} 4$ & $150<\mathrm{Ti} \leq 250$ & \\
$\mathrm{C} 5$ & $\mathrm{Ti}>250$ & \\
\hline
\end{tabular}


Tableau 2: Résultats de l'analyse de variance de la concentration des 159 puits et de chaque zone A et B entre 1993 et 2007.

\begin{tabular}{lccc}
\hline & Entre 1993 et 2003 & Entre 2003 et 2007 & Entre 1993 et 2007 \\
\hline $\begin{array}{l}\text { Analyse statistique } \\
\text { globale (159puits) }\end{array}$ & NS & S & HS \\
\hline $\begin{array}{l}\text { Analyse statistique } \\
\text { Zone A }\end{array}$ & $\mathrm{S}$ & $\mathrm{HS}$ & $\mathrm{HS}$ \\
\hline $\begin{array}{l}\text { Analyse statistique } \\
\text { Zone B }\end{array}$ & $\mathrm{NS}$ & $\mathrm{NS}$ & $\mathrm{S}$ \\
\hline NB : S : Différence significative (à 5\%); NS : Différence non significative ; HS : Différence hautement significative (à 1\%)
\end{tabular}

Tableau 3: Pourcentage du terrain occupé par la pollution nitrique de chaque classe des puits sur le total du terrain cartographié en 1993, 2003 et 2007.

\begin{tabular}{lccccc}
\hline & \multicolumn{5}{c}{ Surface occupée par classe / surface totale $*$ 100 } \\
\cline { 2 - 6 } Année & Classe1 & Classe2 & Classe3 & Classe4 & Classe5 \\
\hline 1993 & $20 \%$ & $27 \%$ & $21 \%$ & $20 \%$ & $12 \%$ \\
2003 & $15 \%$ & $24 \%$ & $22 \%$ & $21 \%$ & $18 \%$ \\
2007 & $11 \%$ & $16 \%$ & $17 \%$ & $31 \%$ & $25 \%$ \\
\hline
\end{tabular}

Tableau 4: Niveaux moyens de la concentration des nitrates, les niveaux Max, Min, et les Ecart-types calculés au niveau des 159 puits en 1993, 2003,2007et 2010.

\begin{tabular}{lcccc}
\hline Paramètre & \multicolumn{4}{c}{ Nitrates $\mathbf{( m g / L )}$} \\
\hline Année & 1993 & 2003 & 2007 & 2010 \\
Valeur Max & 385 & 387 & 679 & 300 \\
Valeur Min & 5,00 & 1,12 & 4,84 & 40 \\
Ecat-type & 73,92 & 81,80 & 109 & 75,15 \\
Moyenne & 107.2 & 120.4 & 146,5 & 150,9 \\
Normes Marocaine & 50 & 50 & 50 & 50 \\
& & & & \\
\hline
\end{tabular}


Tableau 5: Résultats de l'analyse de variance de la concentration des nitrates des 159 puits et de chaque zone A et B entre 1993 et 2010.

\begin{tabular}{lcccccc}
\hline & $\begin{array}{c}\text { Entre } \\
\mathbf{1 9 9 3} \text { et } \\
\mathbf{2 0 1 0}\end{array}$ & $\begin{array}{c}\text { Entre } \\
\mathbf{2 0 0 3} \text { et } \\
\mathbf{2 0 1 0}\end{array}$ & $\begin{array}{c}\text { Entre } \\
\mathbf{2 0 0 7} \text { et } \\
\mathbf{2 0 1 0}\end{array}$ & $\begin{array}{c}\text { Entre } \\
\mathbf{1 9 9 3} \text { et } \\
\mathbf{2 0 0 3}\end{array}$ & $\begin{array}{c}\text { Entre } \\
\mathbf{1 9 9 3} \text { et } \\
\mathbf{2 0 0 7}\end{array}$ & $\begin{array}{c}\text { Entre } \\
\mathbf{2 0 0 3} \text { et } \\
\mathbf{2 0 0 7}\end{array}$ \\
\hline $\begin{array}{l}\text { Analyse statistique } \\
\text { globale (159puits) }\end{array}$ & $\mathrm{HS}$ & $\mathrm{S}$ & $\mathrm{NS}$ & $\mathrm{S}$ & $\mathrm{HS}$ & $\mathrm{HS}$ \\
\hline $\begin{array}{l}\text { Analyse statistique } \\
\text { Zone A }\end{array}$ & $\mathrm{HS}$ & $\mathrm{S}$ & $\mathrm{S}$ & $\mathrm{S}$ & $\mathrm{HS}$ & $\mathrm{HS}$ \\
\hline $\begin{array}{l}\text { Analyse statistique } \\
\text { Zone B }\end{array}$ & $\mathrm{S}$ & $\mathrm{NS}$ & $\mathrm{NS}$ & $\mathrm{NS}$ & $\mathrm{S}$ & $\mathrm{S}$ \\
\hline
\end{tabular}

NB: S : Différence significative ; NS : Différence non significative ; HS : Différence hautement significative

Tableau 6: Pourcentage de la surface occupée par la pollution nitrique de chaque classe des puits sur le total du terrain cartographié en 1993, 2003, 2007 et 2010.

\begin{tabular}{lccccc}
\hline \multirow{3}{*}{ Année } & \multicolumn{5}{c}{ Surface occupée par classe / surface totale *100 } \\
\cline { 2 - 6 } & $\begin{array}{c}\text { Classe1 } \\
(<\mathbf{5 0} \mathbf{~ m g} / \mathbf{L})\end{array}$ & $\begin{array}{c}\text { Classe2 } \\
(\mathbf{5 0 - 1 0 0} \\
\mathbf{m g} / \mathbf{L})\end{array}$ & $\begin{array}{c}\text { Classe3 } \\
(\mathbf{1 0 0 - 1 5 0} \\
\mathbf{m g} / \mathbf{L})\end{array}$ & $\begin{array}{c}\text { Classe4 } \\
(\mathbf{1 5 0 - 2 5 0} \\
\mathbf{m g} / \mathbf{L})\end{array}$ & $\begin{array}{c}\text { Classe5 } \\
\mathbf{2 5 0} \\
\mathbf{m g} / \mathbf{L})\end{array}$ \\
\hline 1993 & $20 \%$ & $27 \%$ & $21 \%$ & $20 \%$ & $12 \%$ \\
2003 & $15 \%$ & $24 \%$ & $22 \%$ & $21 \%$ & $18 \%$ \\
2007 & $11 \%$ & $16 \%$ & $17 \%$ & $31 \%$ & $25 \%$ \\
2010 & $07 \%$ & $12 \%$ & $11 \%$ & $18 \%$ & $52 \%$ \\
\hline
\end{tabular}

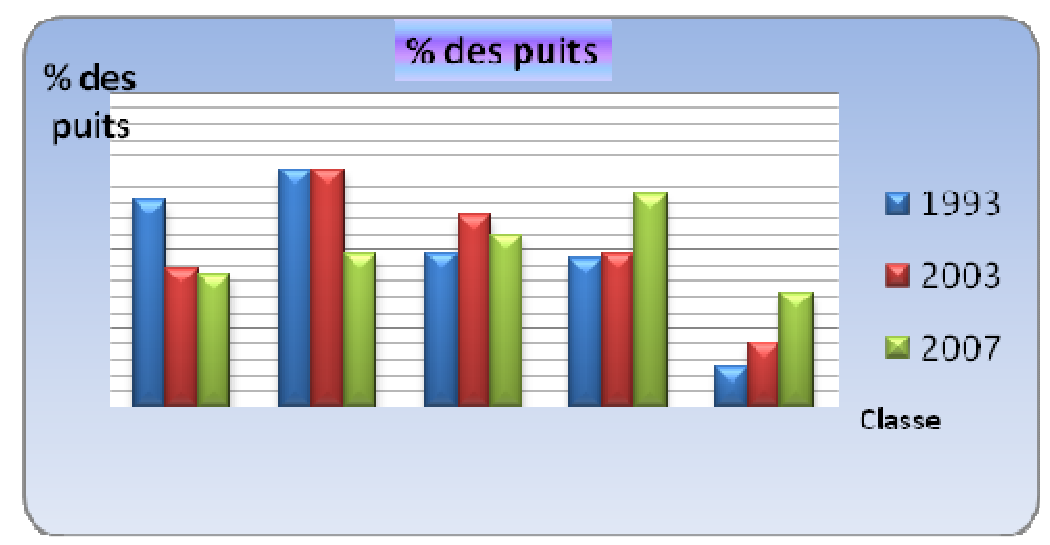

Figure 5: Variation spatiotemporelle du pourcentage des puits selon la concentration des nitrates des eaux des 159 puits des M'nasra en 1993, 2003 et 2007. 


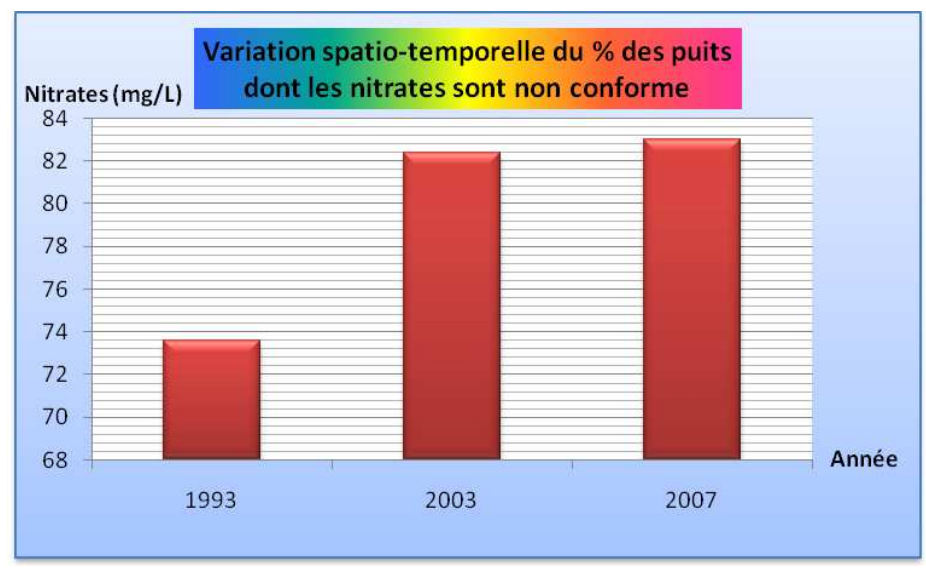

Figure 6: Variation spatio-temporelle du pourcentage des puits possédant une concentration en nitrates supérieure à $50 \mathrm{mg} / \mathrm{L}$.

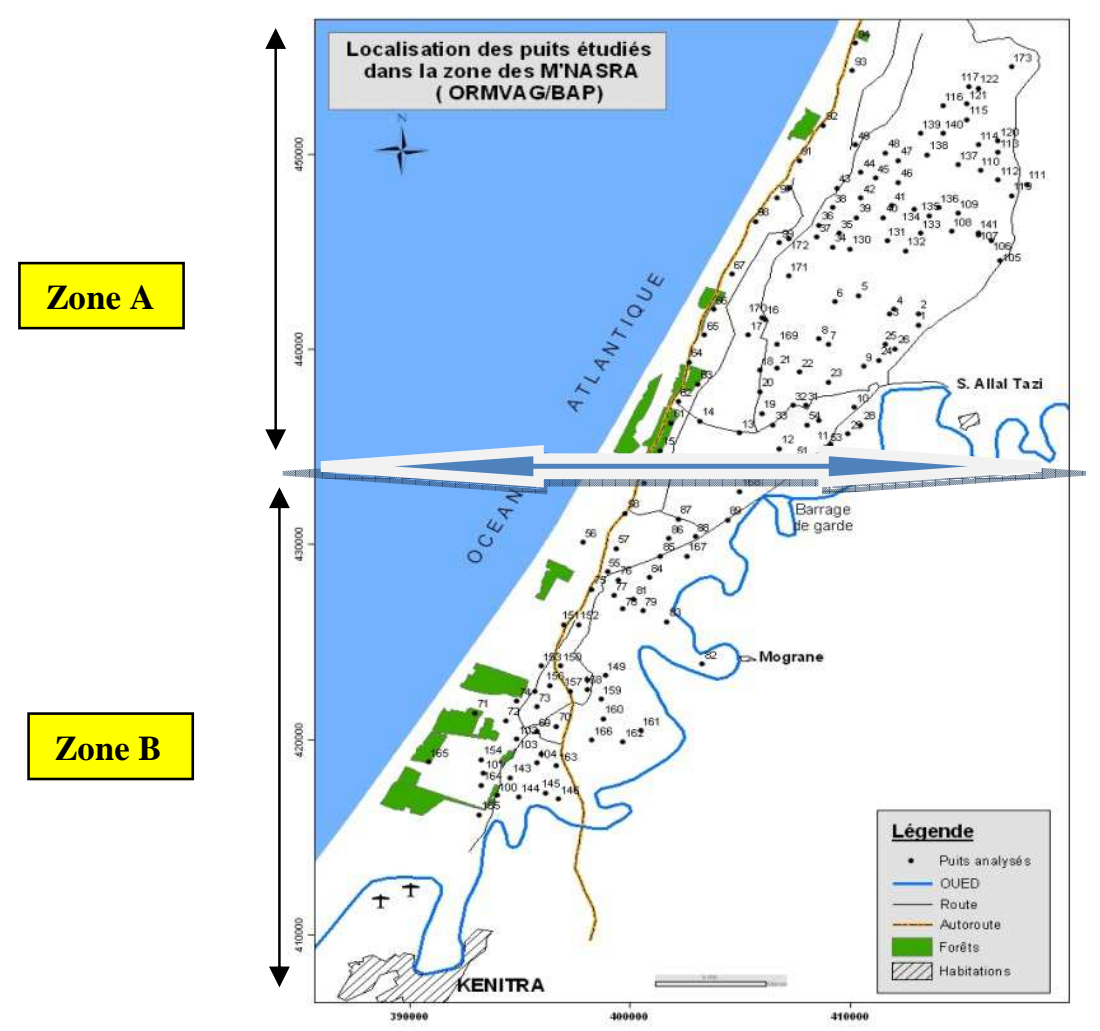

Figure 7: Carte de la situation des 159 puits étudiés dans la zone M’nasra (Zone A+ Zone B). 


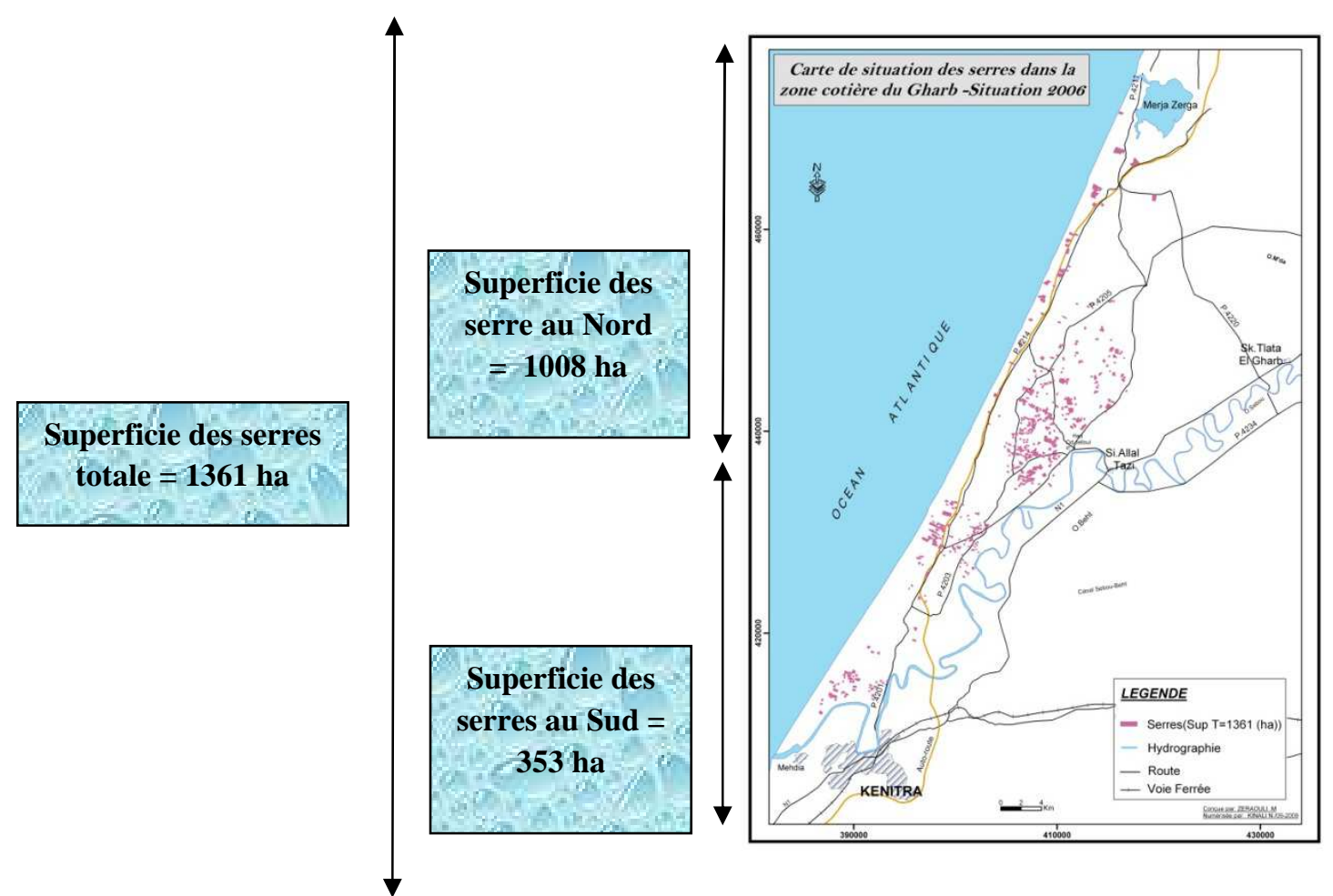

Figure 8: Carte de la situation des serres dans la zone côtière du Gharb (ORMVAGDDA /SEA/BAP).

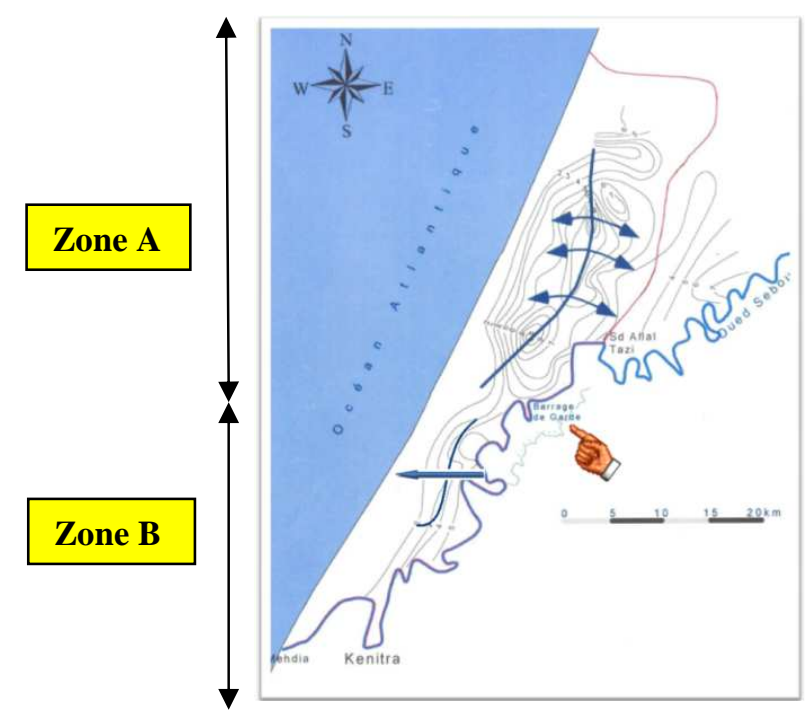

Figure 9: Carte piézométrique présentant les directions d'écoulements souterraine de la zone A et B de la nappe phréatique de M'nasra. 


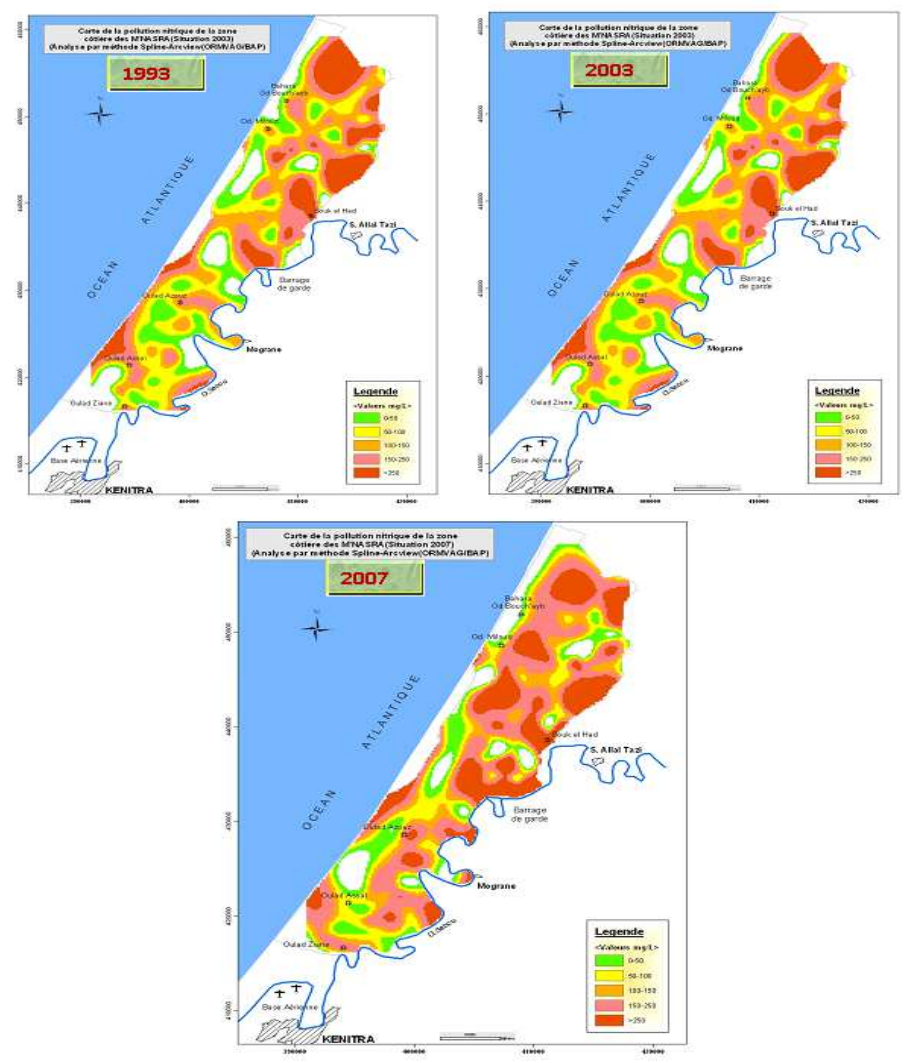

Figure 10: Carte de la pollution nitrique de la zone de M'nasra, Analyse par méthode Spline Arcview Situation 1993,2003 et 2007.

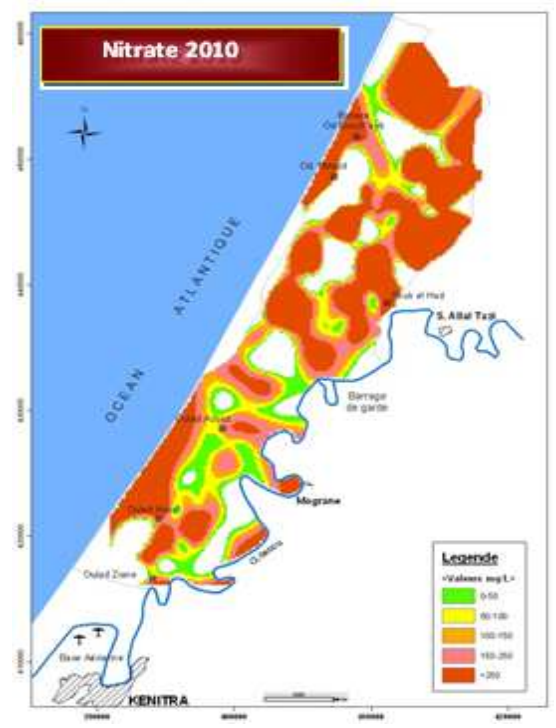

Figure 11: Carte de la pollution nitrique de la zone de M'nasra, Analyse par méthode Spline Arcviw Situation 2010. 

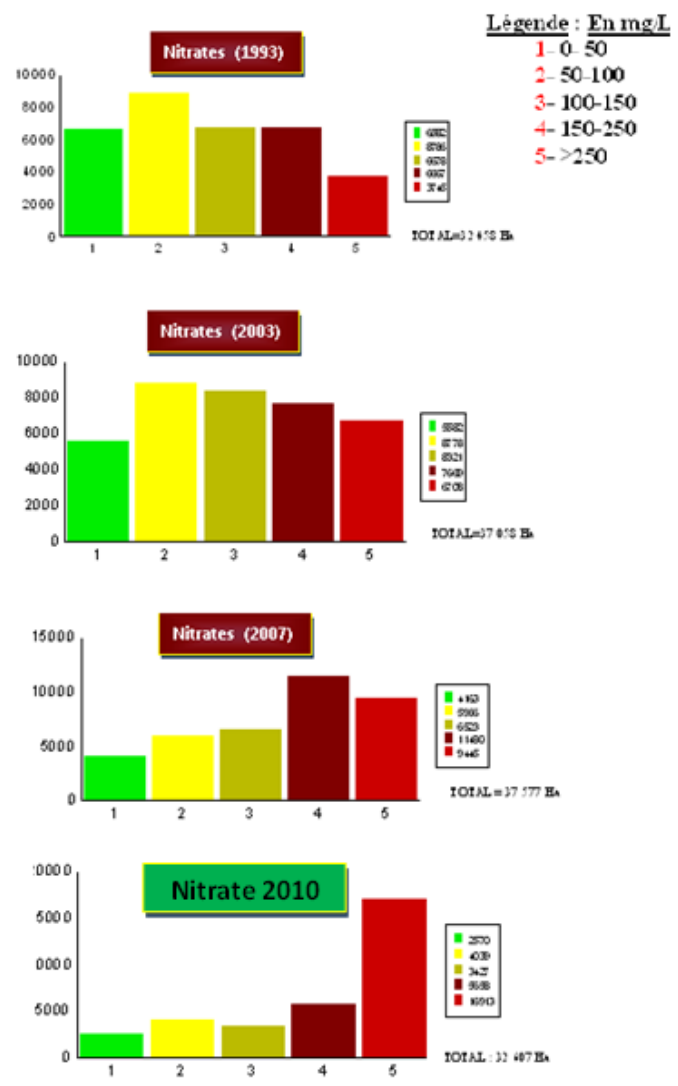

Figure 12 : Répartition spatiales des cinq classes des concentrations des nitrates en 1993, 2003, 2007 et 2010 .

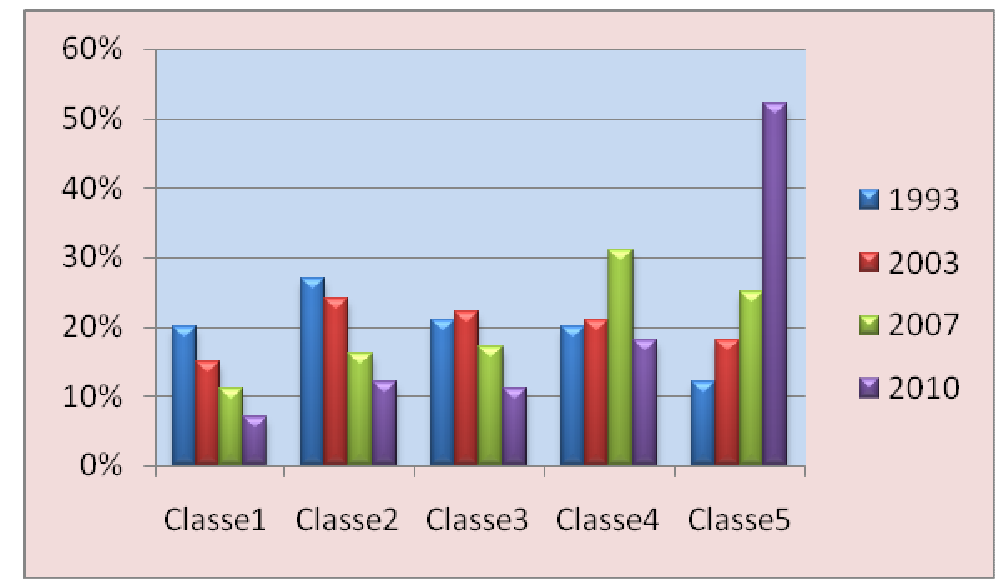

Figure 13 : Variation spatio-temporelle du pourcentage de terrain occupé par chaque classe de la concentration des nitrates $(1993,2003,2007$ et 2010). 


\section{Conclusion}

La pollution diffuse, causée par l'application de fertilisants sur les parcelles agricoles, entraîne une dégradation de la qualité de l'eau souterraine. L'azote est le fertilisant le plus difficile à gérer en agriculture.

Les résultats obtenus ont montré une pollution azotée évolutive et répartie. En effet, $80 \%$ des puits prospectés et analysés ne répondent pas aux normes de potabilité. Par ailleurs, l'analyse spatiale a révélé une pollution nitrique d'une superficie de $7538 \mathrm{Ha}$ enregistrée entre les périodes de campagnes d'échantillonnage en 1993 et en 2007.

En revanche, après les pluies exceptionnelles de la période de compagnie 2009/2010 (746 mm), on a pu observer une dégradation de la qualité de la nappe aussi bien au niveau ponctuel qu'au niveau spatial, surtout dans la partie Nord de la zone étudiée (au nord de la ligne de cordonnée Y $=430000$ $\mathrm{m})$, soit d'une superficie avoisinant 2076 ha.

\section{REFERENCES}

Afnor NFT. 1977. Mesure de la conductivité et de la température. Association Française de Normalisation.

Boudjadja A, Messahel M. 2003. Ressources hydriques en Algérie du Nord. Sci. Eau., 16(3): 285-304.

Bricha S, Ounine K, Oulkhir S, Elhaloui N, Atrassi B. 2007. Etude de la qualité physicochimique et bactériologique de la nappe phréatique M'nasra (Maroc). Afriq. Sci ., 03(3): 391-404.

DPA. 2006. Rapports bilans et activités M'nasra, Maroc.

DRPE (Direction de la Recherche et de la Planification des Eaux). 1994. Etude de modélisation de la nappe côtière du Gharb (Région du M'nasra).

DRPE (Direction de la Recherche et de la Planification des Eaux). 2006. Etude de modélisation de la nappe côtière du Gharb (Région du M'nasra).

Goula BTA, Kouame IK, Coulibaly L,
Gnagne T, Savane I, Djoman PD. 2007. Caractérisation des effluents de boues activées d'une usine agroalimentaire en vue de son utilisation comme eau d'arrosage de pelouses en zone tropicale humide. Sci. Eau., 20(3): 299-307.

Hatira A, Baccar L, Grira M, Gallali T. 2007. Analyse de sensibilité du système oasien et mesure de sauvegarde de l'oasis de Métouia. Sci. Eau., 20(1): 59-69.

Ibnoussina M, El Halui M, Maslouhi A. 2006. Expérimentation et modélisation de la lixiviation de l'Azote nitrique dans le sol sableux. C.R. Géoscience, 338: 787-794.

Idrissi AI, Rhidouani A, Zeraouli M, Echchelh A, Addou M, Ben AS. 2011.spatial and temporal study of the salinization quality of groundwater: case of m'nasra (morocco). Phys. Chem. News, 58: 25-29.

Idrissi AI, Zeraouli M, Addou M, Soulaymani A, Rhidouani A. 2009. Évaluation de la pollution nitrique de la nappe phréatique de la zone côtière du Gharb (M'nasra) au Maroc entre 1993 et 2007. Gestion des Produits Chimiques pour un Meilleur Respect de l'Environnement Proceedings GEPORE3., 1(18): 10-18.

Idrissi AI, Rhidouani A, Zeraouli M,Haidar A, Echchelh A, Addou M. 2010.Spatial and temporal study of the physical and chemical quality of groundwater: Case of M. nasra (Morocco). Environmental Science., 5(1): 51-58.

Idrissi AI, Zeraouli M, Addou M, Moukhtari A, Soulaymani A. 2007.Évaluation de la pollution nitrique de la nappe phréatique de la zone côtière du Gharb (M'nasra) au Maroc entre 1993 et 2003. Afriq. Sci., 3(3): 378-390.

Idrissi AI, Rhidouani A, Zeraouli M,Haidar A, Echchelh A, Addou M. 2010. Sodization, a threat to coastal irrigated areas case ofM.nasra (Morocco). Environmental Science., 5(1): 46-50.

Laurence Mir. 2007. Nitrates dans l'eau de boisson et risque de décès par cancer de 
la vessie à Taïwan. Environnement, Risques \& Santé, 6(5) : 337-8.

Laurianne G. 2008. TNFA locus is associated with $\beta^{\circ} 39$ thalassemia in Corsica and Sardinia. European Cytokine Network, 19(4): 196-203.

Leclerc H. 2008. Nitrates de l'eau d'alimentation et risques pour la santé. Comptes rendus de l'Académie d'Agriculture de France. CODEN CRAFEQ, 94(3): 14-24.

Lenntech. 2008. Traitement de l'Eau et de l'Air. Rotterdamseweg 402 M: France Belgique - Suisse - Canada - Pays Bas.

L'hirondel JL. 2002. Nitrate and Man: Toxic, Harmless or Beneficial ? CABI Publishing : New York ; 168 pp.

Little AD. 1971. Inorganic chemical pollution of freshwater. Environmental Protection Agency: Washington, DC, U.S.

Lhadi EK, Mountadar M, Younsi A. 1993. Contamination des eaux souterraines par les sels et les nitrates dans la zone comprise entre Azemmour et Bir Jdid (Maroc). Séminaire Recherche. Nation Eau. Et Envir. LPEE. Casablanca, Maroc.

ORMVAG. 2010. Impact des inondations enregistrées en 2009/2010 sur le secteur agricole au niveau de la Région Gharb

Chrarda Beni Hssen (Premières estimations).

Resencement Général de la Population. 2004. communes Rurales de M'nasra et Ben Mansour.

Rodier J. 1984. L'Analyse de l'Eau: Eaux Naturelles, Eaux Résiduaires, Eaux de $\operatorname{Mer}\left(8^{\text {ème }}\right.$ edn). Dénod : Paris ; 1383p.

Scet-Maroc. 1999. Projet de Protection de l'Environnement du bassin de Sebou (PPBS). Etude pour un programme d'action visant à minimiser et à contrôler l'impact des engrais et des pesticides sur l'environnement du bassin de Sebou. Sous-mission 2.1. Secrétariat d'Etat Chargé de l'Environnement.

Younsi A. 1994. Etude de mécanisme des intrusions marines vers le système aquifer compris entre Oued Oum Rbia et Bir Jdid. Thèse Univ., El jadida, Maroc,147p.

Zeraouli M, Morchid M. 2001. Fertilisation azotée du poivron sous tunnel dans une zone sableuse a nappe proche de la surface. Marocaine des Sci. et Tech. du Développement Rural, 31(118). 